ABSTRACT: Reproducible neurophysiologic testing paradigms are critical for multicenter studies of neuropathy associated with impaired glucose regulation (IGR), yet the best methodologies and endpoints remain to be established. This study evaluates the reproducibility of neurophysiologic tests within a multicenter research setting. Twenty-three participants with neuropathy and IGR were recruited from two study sites. The reproducibility of quantitative sudomotor axon reflex test (QSART) and quantitative sensory test (QST) (using the CASE IV system) was determined in a subset of patients at two sessions, and it was calculated from intraclass correlation coefficients (ICCs). QST (cold detection threshold: ICC $=0.80$; vibration detection threshold: ICC $=0.75$ ) was more reproducible than QSART (ICC foot $=0.52$ ). The performance of multiple tests in one setting did not improve reproducibility of QST. QST reproducibility in our IGR patients was similar to reports of other studies. QSART reproducibility was significantly lower than QST. In this group of patients, the reproducibility of QSART was unacceptable for use as a secondary endpoint measure in clinical research trials.

Muscle Nerve 39: 529-535, 2009

\title{
RELIABILITY OF QUANTITATIVE SUDOMOTOR AXON REFLEX TESTING AND QUANTITATIVE SENSORY TESTING IN NEUROPATHY OF IMPAIRED GLUCOSE REGULATION
}

\author{
AMANDA PELTIER, MD, ${ }^{1}$ A. GORDON SMITH, MD, ${ }^{2,3}$ JAMES W. RUSSELL, MD, MS, ${ }^{4}$ KIRAN SHEIKH, MD, ${ }^{5}$ \\ BILLIE BIXBY, BS, ${ }^{2}$ JAMES HOWARD, BS, ${ }^{2}$ JONATHAN GOLDSTEIN, MD, ${ }^{6}$ YANNA SONG, MS, ${ }^{7}$ LILY WANG, PhD, ${ }^{7}$ \\ EVA L. FELDMAN, MD, PhD, ${ }^{5}$ and J. ROBINSON SINGLETON, MD ${ }^{2}$ \\ ${ }^{1}$ Department of Neurology, Vanderbilt University, Nashville, Tennessee, USA \\ ${ }^{2}$ Department of Neurology University of Utah, Salt Lake City, Utah, USA \\ ${ }^{3}$ Department of Pathology, University of Utah, Salt Lake City, Utah, USA \\ ${ }^{4}$ Department of Neurology, University of Maryland and Maryland VA Healthcare System, 22 South Greene Street, \\ Box 175, Baltimore, Maryland 21201-1595, USA \\ ${ }^{5}$ Department of Neurology, University of Michigan, Ann Arbor, Michigan, USA \\ ${ }^{6}$ Department of Neurology, Yale University School of Medicine, New Haven, Connecticut, USA \\ ${ }^{7}$ Department of Biostatistics, Vanderbilt University, Nashville, Tennessee, USA
}

Accepted 24 September 2008

\begin{abstract}
A need exists for accurate and early diagnosis of neuropathy in clinical trials where participants have impaired glucose regulation [impaired fasting glucose (IFG) and/or impaired glucose tolerance (IGT)]. Neuropathy trials require screening tests that are sen-
\end{abstract}

\footnotetext{
Abbreviations: ADA, American Diabetes Association; CDT, cold detection threshold; CASE IV, computer-aided sensory evaluator system; HIV, human immunodeficiency virus; IFG, impaired fasting glucose; IGT, impaired glucose tolerance; ICC, intraclass correlation coefficient; IENFD, intraepidermal nerve fiber density; IGR, impaired glucose regulation (includes IGT, IFG); JND, just noticeable difference (discrete magnitudes of stimulation by the CASE IV exponential algorithmic function used to determine threshold of perception using CASE IV): NCS, nerve conduction studies; QSART, quantitative sudomotor axon reflex test; QST, quantitative sensory test; SND, standard norma deviate; VDT, vibration detection threshold; WHO, World Health Organization Key words: clinical trials; impaired glucose tolerance; neuropathy; QSART; QST
}

Correspondence to: J.W. Russell; e-mail: jrussell@som.umaryland.edu

(c) 2009 Wiley Periodicals, Inc.

Published online 15 March 2009 in Wiley InterScience (www.interscience. wiley.com). DOI 10.1002/mus.21210 sitive, specific, can be performed without highly specialized training, and are standardized across multiple study sites. The ideal test would allow rapid and reproducible screening of potential participants. When used as a primary or secondary endpoint measure in longitudinal studies, the test needs to be reproducible to ensure that variations in results are due solely to changes in nerve physiology. A further problem facing investigators in large clinical neuropathy trials is the choice of an appropriate primary endpoint measure. Electrophysiologic tests offer the advantage of early preclinical diagnosis when early intervention may prevent neuropathy. However, nerve conduction studies (NCS), which are the most commonly used electrophysiologic tests, do not evaluate small fiber function and thus they may fail to identify patients with this type of neuropathy.

Quantitative sensory testing (QST) and quantitative sudomotor axon reflex testing (QSART) have 
both been studied in large, single-center studies of diabetic neuropathy. ${ }^{7,8,10}$ Within the expertise of a single site, QST has been shown to provide highly reproducible within-test and between-test results. ${ }^{1,7,10,12,13,16,26}$ QST has been used as a secondary endpoint in a number of clinical studies that evaluated therapeutic outcomes, such as human immunodeficiency virus (HIV)-related and diabetic neuropathies, $, 311,39$ and QSART has been used as a confirmatory test of neuropathy in several small studies of small fiber neuropathy. ${ }^{21,27,31,33}$ The reliability and reproducibility of QSART has not been evaluated in a multicenter trial, nor has its reproducibility been compared with other measures of small fiber function. Evaluation of the reproducibility of these tests in the setting of a multicenter clinical trial is important in promoting effective use of neurophysiologic tests as surrogate endpoint measures of neuropathy in future clinical trials.

\section{METHODS}

Study Design. Participants enrolled in a non-randomized longitudinal cohort study evaluating early neuropathy associated with IGR (as part of the Impaired Glucose Tolerance Causes Neuropathy Study, NIH NS38849) at three centers. Patients were evaluated with nerve conduction studies, QST [vibration detection threshold (VDT) and cold detection threshold (CDT)], QSART, and intraepidermal nerve fiber density (IENFD) through skin biopsy as part of the study design. The criteria for inclusion within the study were: IGR confirmation on at least two separate occasions; signs and symptoms of peripheral neuropathy; and an abnormality in at least one of the following: nerve conduction studies (NCS), QST, or QSART. Symptoms and signs of neuropathy included numbness, paresthesias, and/or pain in the extremities in a stocking/glove distribution; absent or reduced reflexes; and decreased perception of vibration, sharp stimuli, or proprioception on examination. IGR was defined according to the World Health Organization (WHO) and American Diabetes Association (ADA) definition at that time: fasting glucose between 110 and $126 \mathrm{mg} / \mathrm{dl}$, and/or 2-hour glucose between 140 $\mathrm{mg} / \mathrm{dl}$ and $200 \mathrm{mg} / \mathrm{dl}^{16,36}$

IENFD was not chosen as an initial screening measure, because one purpose of the study was to pilot its use as an endpoint. To allow entry based on its abnormality would have biased the results in its favor. Subjects were excluded from the study if other causes of neuropathy existed. Exclusions included concurrent use of neuropathy-inducing medication, exposure to environmental toxins, hereditary neuropathy, and abnormal laboratory screening results (thyroid-stimulating hormone, serum protein electrophoresis and immunofixation, antinuclear antibody, vitamin $\mathrm{B}_{12}$ level, folate level, and erythrocyte sedimentation rate). Subjects provided consent according to the Declaration of Helsinki. Studies were performed sequentially on participants from two study sites, the University of Michigan and the University of Utah. Reproducibility testing for QST and QSART was performed on two separate days separated by intervals of at least 1-30 days in a subset of 23 patients.

Patients from two of the three centers participating in the study were included in the reproducibility study. Of the 23 total patients included, 19 patients underwent repeat QST testing, and 13 patients underwent repeat QSART testing. Nine patients had both QSART and QST repeat testing, 10 had only repeat QST testing, and 4 had only repeat QSART testing. This was due to subject availability for repeat testing, and therefore not all subjects were able to have both QST and QSART repeat testing performed. These 23 patients were not significantly different from the rest of the cohort evaluated and were the initial patients enrolled in the IGT cohort.

QST. QST was performed on the left dorsal foot using the computer-aided sensory evaluator system (CASE IV; WR Medical Electronics, Stillwater, Minnesota) and previously published methodology. ${ }^{11,37}$ Vibration and cold detection thresholds (VDT and CDT) were tested consecutively in each patient. Subjects received three tests each of VDT and CDT on day 1 (trial 1) and day 2 (trial 2). Three tests were performed at each session to determine whether frequent testing improved the reproducibility of results. The examiner read the instructions at the beginning of each trial to ensure that the participant fully understood the instructions. Conditions of testing were standardized and remained identical between individual tests and trials.

QSART. QSART was performed only once on each day, because residual sweat volume and local skin alterations can cause variability of results. ${ }^{26}$ The skin was prepped with acetone, isopropyl alcohol, and water. Capsules were placed on the medial forearm (75\% of the distance from the ulnar epicondyle to the pisiform bone), the proximal leg (lateral aspect, $5 \mathrm{~cm}$ distal to the fibular head), medial distal leg (5 $\mathrm{cm}$ proximal to the medial malleolus), and proximal foot (over the extensor digitorum brevis muscle). Ten percent acetylcholine was iontophoresed for 5 


\begin{tabular}{|c|c|c|c|c|}
\hline Measure & CDT test 1 & CDT test 4 & VDT test 1 & VDT test 4 \\
\hline $\begin{array}{l}\text { Mean JND } \\
\text { Mean approximate change from } \\
\text { baseline }\left({ }^{\circ} \mathrm{C} \text { for CDT or } \mu \mathrm{m}\right. \\
\text { for VDT) } \\
\text { ICC }\end{array}$ & $16.9 \pm 4.73$ & $16.20 \pm 5.17$ & $\begin{array}{r}172.5 \\
0.75\end{array}$ & $\begin{array}{l}120 \\
0.75\end{array}$ \\
\hline Measure & CDT trial 1 & CDT trial 2 & VDT trial 1 & VDT trial 2 \\
\hline $\begin{array}{l}\text { Mean JND } \\
\text { Mean approximate change from } \\
\text { baseline }\left({ }^{\circ} \mathrm{C} \text { for CDT or } \mu \mathrm{m}\right. \\
\text { for VDT) } \\
\text { ICC }\end{array}$ & $16.23 \pm 5.18$ & $15.85 \pm 5.63$ & $21.32 \pm 1.94$ & $20.64 \pm 3.45$ \\
\hline
\end{tabular}

JND, just noticeable difference (discrete magnitudes of stimulation by the CASE IV device exponential algorithmic function); CDT, cold detection threshold; VDT, vibration detection threshold; ICC, intraclass correlation coefficient. Approximate change from baseline is mean of minimum degrees of temperature/ $\mu m$ vibration required for patients to perceive stimulus from baseline temperature/vibration at beginning of CASE IV testing paradigm.

minutes. The sweat response was recorded over 10 minutes ( 5 minutes of ionotophoresis plus 5 minutes for sweat volumes to return to baseline). QSART and CASE IV equipment were purchased from WR Electronics (Stillwater, Minnesota). They are similar but not identical to the original equipment designed at the Mayo Clinic and are used in clinical trials at that center.

Statistical Design. Intraclass correlation coefficients (ICCs) were calculated to investigate the reliability of the QST and QSART scores. Each test was considered to be a random sample of all possible tests so that the results could be generalized..$^{40}$ SAS macro \%INTRACC (http://support.sas.com/kb/ 25/031.html) was used to calculate ICCs. Because of the nonlinear aspect of patient sensation expressed in just-noticeable-difference (JND) values, the normal deviate was also used for analysis of QST results, but there was no significant difference in ICC values calculated from JND or normal deviates. CDT and VDT were analyzed separately. ICC for QSART results were calculated using both raw sweat volumes and standard normal deviates (SNDs). Standard normal deviates (z-score) for QSART sweat volumes were adjusted to compare with previously published normative data. ${ }^{26}$ This did not produce significantly different results, and therefore the ICC results reported are from raw sweat volumes.

Analyses were performed using SAS, version 9.1.3 (SAS Institute, Inc., Cary, North Carolina) and R, version 2.3.1.

\section{RESULTS}

For analysis of the reproducibility of neurophysiologic tests, 23 subjects were included prospectively in the dataset. The average age of the cohort was 57.6 years (range $45-74$ years). There were 7 males and 16 females, among whom 1 was African American, 2 Hispanic, and the remainder Caucasian. The mean body mass index was 32.7 (range 25.3-38.7). Hemoglobin $\mathrm{A}_{1 \mathrm{C}}$ levels fell within the normal range on tested participants. Patients had an average neuropathy duration of 60 months before being enrolled in the study (range 12-180). The average fasting glucose was 99, and the 2-hour glucose was $166 \mathrm{mg} / \mathrm{dl}$ $(142-199 \mathrm{mg} / \mathrm{dl})$. Nineteen of 23 subjects participated in QST reproducibility testing, and 13 of 23 completed QSART in both sessions. Nine of 23 participants completed both QST and QSART and are included in both results.

Reproducibility of CDT and VDT Results. Comparison of test 1 (first test of trial 1) and test 4 (first test of trial 2) yielded an ICC of 0.81 for CDT (Table 1). Utilizing the means of tests on trial 1 and trial 2 did not improve reliability significantly (ICC 0.83). Reproducibility between tests within each trial is shown in Figure 1B, and it was not as strong; the ICC for test 1 vs. $2(0.83)$ vs. $1-3$ was 0.85 , and thus there was no improvement in reliability between trial 2 and 3 compared with 1 and 2.

VDT reproducibility was similarly strong between trials. The ICG for VDT test 1 and test 4 was 0.74 (Table 1). The results were not significantly different for means of VDT tests of trial 1 and trial 2 (ICC 0.82 ) as indicated in Figure 1A. Reproducibility of individual tests performed on the same day was significantly lower for VDT than for CDT (0.82 vs. $0.55)$. One patient performed with more variability on the second day, which may have led to the significantly lower ICC mean of trials for VDT. 

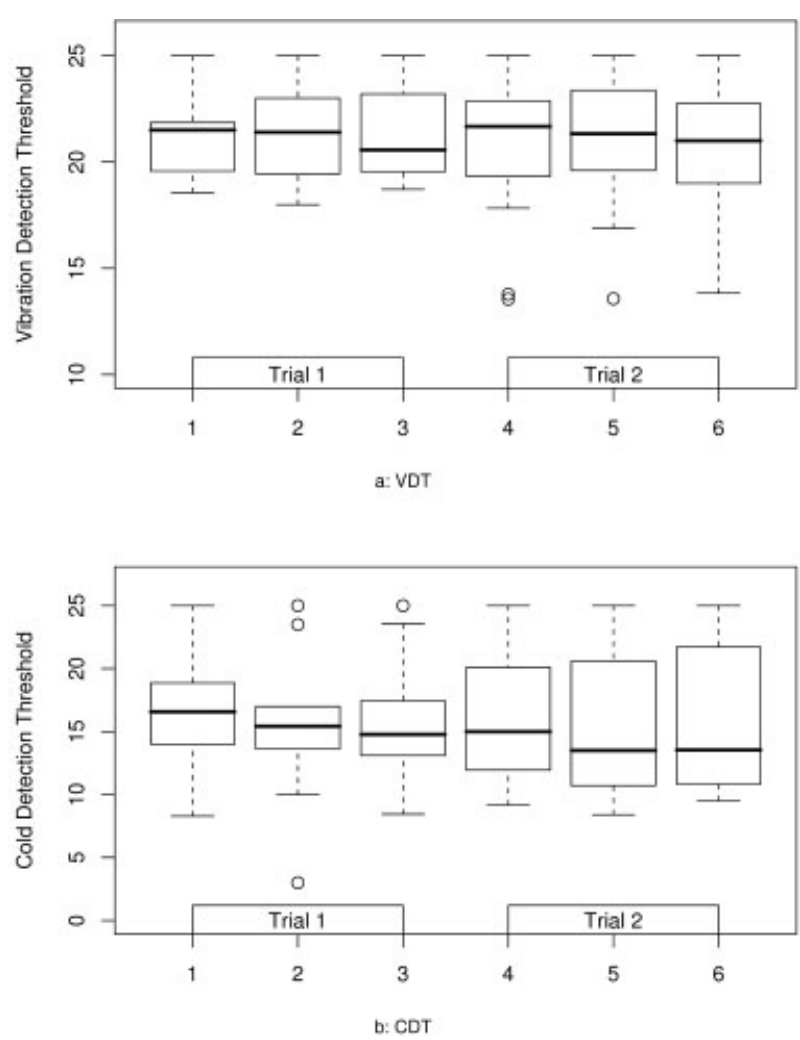

FIGURE 1. (A) Reproducibility of VDT between individual tests $(T 1-6)$ and trials in subjects with impaired glucose tolerance and small fiber neuropathy. The boxplot indicates the mean, 25th and 75th percentiles for VDT expressed in JND units for each test. (B) Reproducibility of CDT between individual tests (T1-6) and trials in subjects with IGR and neuropathy. The boxplot indicates the mean, 25th and 75th percentiles for CDT results expressed in JND units for each test.

Reproducibility of QSART Results. No patient had a lack of sweat response at all four sites. Most participants had a proximal-to-distal gradient, with higher sweat volumes proximally. Reproducibility was similar for the forearm, proximal leg, and foot sites (0.52-0.63; Table 2). The distal leg site was the least reproducible, with an ICC of 0.42 . One patient had a severe leak at the forearm and proximal leg sites. It was impossible to accurately determine the volume at these sites, and the data were excluded from the analysis.

\section{DISCussion}

Neuropathy occurs over time in more than half of diabetic patients. ${ }^{11,49}$ Nerve conduction studies demonstrated that neuropathy is already present in $10-$ $18 \%$ of patients at the time of diabetes diagnosis, ${ }^{6,23}$ suggesting that peripheral nerve injury occurs in the early stages of disease and with milder degrees of glycemic dysregulation. Neuropathy that occurs early in diabetes is usually characterized by symmetrical sensory symptoms including pain and autonomic dysfunction. ${ }^{32,38,41,44,45,47}$ In the largest prospective series, $81 \%$ of neuropathy patients with IGT had exclusively sensory complaints, and $92 \%$ recognized neuropathic pain as a dominant symptom of their neuropathy. ${ }^{41}$ Small myelinated and unmyelinated fibers convey sensations of light touch, pain, and temperature, ${ }^{2,18}$ whereas large fiber loss results in decreased vibratory sensation, joint position sense, and sural sensory nerve action potentials (SNAPs). ${ }^{29,37}$ Pain symptoms and impaired temperature perception in IGT neuropathy suggest prominent early involvement of the small unmyelinated nerve fibers that mediate pain, temperature sensation, and autonomic function. However, the present study indicates that, in IGT neuropathy, as in diabetic neuropathy, large myelinated fibers are also affected as determined by an abnormal VDT in up to $72 \%$ of participants (using the 95 th percentile) and an abnormal sural SNAP amplitude in $76 \%$ of participants. ${ }^{46}$ This indicates that there is significant involvement of distal large fiber function even before a patient converts from IGT or IFG to diabetes. Furthermore, these findings support the concept that tests of large fiber function should be included in trial entry criteria for early diabetic neuropathy.

Identification of ideal neuropathy endpoints has been controversial. Recently, a case definition of distal symmetrical polyneuropathy was developed to standardize clinical research. ${ }^{14}$ This report concluded that simple composite examination scores were as accurate as more complex examinations. However, QST and autonomic tests were not included in the recommendations because of concerns

Table 2. Quantitative sudomotor axon reflex test (QSART): means of trial 1 vs. trial 2.

\begin{tabular}{llll}
\hline Measure $(\mu l)$ & Trial 1 & Trial 2 & ICC \\
\hline Mean sweat volume at the foot & $0.76 \pm 0.31$ & $0.76 \pm 0.19$ & 0.52 \\
Mean sweat volume at the distal leg & $1.24 \pm 0.31$ & $1.83 \pm 0.74$ & 0.42 \\
Mean sweat volume at the proximal leg & $0.76 \pm 0.19$ & $0.98 \pm 0.20$ & 0.63 \\
Mean sweat volume at the forearm & $1.17 \pm 1.35$ & $0.73 \pm 0.73$ & 0.55 \\
\hline
\end{tabular}

ICC, intraclass correlation coefficient. 
about the variability of QST or the lack of availability of autonomic testing at medical centers.

The CASE IV equipment used in this multicenter study is now available at many medical centers that conduct neuropathy trials, and it is familiar to those carrying out diabetic neuropathy trials.

Reproducibility results for QST using the CASE IV system in this multicenter study are similar to those reported in other multicenter evaluations. ${ }^{4,22}$ The slightly lower results may be a reflection of sample size, or they could be due to the patient sample. Previous studies of QST reproducibility were performed in patients with diabetic neuropathy, which was most likely more severe than our cohort. This may have led to more patients with QST results at the upper end of the threshold scale (close to the 99th percentile cutoff for JND), which would have minimized the variability and the dynamic range of the test in these cohorts.

QST can measure multiple stimuli thresholds, but it has the drawback of being a biopsychophysical test, and it has been argued that QST is limited by the subject's motivation and concentration during testing. ${ }^{15}$ Despite provisions made in Case IV QST testing for such an eventuality, a subject may subconsciously influence results of the test and make QST unreliable. ${ }^{9,15}$ However, in clinical trials where participants are motivated to closely follow the testing protocol, and where such a profound conscious bias is lacking, this study indicates that QST would be a reliable test.

There are multiple commercially available QST methodologies. Most QST testing uses either the method of limits or the method of levels. The method of limits uses a continuously increasing or decreasing stimulus, and patients report when they feel the stimulus, or the stimulus is absent. This method (because it depends on subject reaction time) is dependent on patient motor abilities and attention. The method of levels ("forced choice") utilized in the Case IV algorithm uses a series of predefined amounts of stimulus, and patients report whether or not they perceive the sensation. The stimulus is increased or decreased based on the patient's choice. This method is more time-consuming, but it may be more accurate due to its independence from reaction time. If a patient makes a choice twice that is incompatible with previous choices, the testing stops.

Other QST systems have been evaluated for reliability, including the vibraton, neurothesiometer, biothesiometer, the Rydel-Seiffer tuning fork for vibration perception thresholds, Medoc, and the Marstock device, among others. However, compari- son between instruments is difficult due to multiple statistical analyses used for evaluation of variability. The neurothesiometer and Reidel-Seiffer tuning fork have also been shown to have significant reproducibility, with coefficients of variance of $8-13 \%$ and $4 \%$, respectively. ${ }^{5,19}$ Reliability of thermal thresholds appears to be less than for vibration perception thresholds when the Medoc device is used, but this was not seen in this study utilizing the CASE IV system. ${ }^{28}$ QST testing may also be more reliable than current perception threshold (CPT) testing. ${ }^{28}$

In our study, we compared variability between separate days using both a single test (the first test on each day) and the mean of three tests performed on the same day, as well as variability between tests performed on the same day. Both CDT and VDT testing were performed similarly by comparing the first trial on each session as well as the mean of each session. There was a significant difference between the two tests in intra- and intrasession variability, as VDT performed less well when the mean of each session was used. This may have been due to the small sample size. Results otherwise did not improve or decrease in reliability between test 1 and 2, and between test 2 and 3 . This suggests that subject conditioning or distractibility were not consistent factors in causing changes in results between individual tests. The relatively high reproducibility obtained in this multicenter study will increase the statistical power for QST as an endpoint measure compared with other less reproducible electrophysiologic measures. However, these results, especially for VDT, suggest that multiple tests at one session may be deleterious with regard to reproducibility.

QSART is an objective measure of postganglionic sudomotor function, which is typically involved in small fiber neuropathies and autonomic neuropathies. ${ }^{26,35,42}$ Unlike QST, QSART permits comparison of distal to proximal sweat responses and thus assessment of length-dependent neuropathies. QSART has relatively good sensitivity in this population, ${ }^{40}$ and it is also abnormal in patients with IGR without defined neuropathy compared with normoglycemic patients. This suggests it has sensitivity in identification of preclinical neuropathy in this population. ${ }^{17}$ However, QSART reproducibility was significantly lower than that for QST, mitigating its usefulness as a longitudinal measure in future research trials.

The ICC values for QSART in this study indicate that reproducibility of QSART may be inadequate in a large clinical trial. This is most likely due to using iontophoresed acetylcholine to induce the sweat response. This increases the number of steps required 
to induce the sweat response as well as the number of variables that can affect QSART results, such as skin temperature, the concentration and purity of acetylcholine solution used, possible air bubbles, ineffective skin preparation, variable skin impedance, or variability in the stimulator output. These variables may have caused the low reproducibility reported in this study. In addition, the commercially available QSWEAT device was used at both sites, whereas previous reproducibility data for QSART were obtained at the Mayo Clinic with similar but not identical equipment. The differences between the two devices may also have contributed to the decreased reproducibility observed. However, the current study clearly indicates that future multicenter studies using available equipment should expect only moderate reproducibility in the QSART, and this will significantly reduce the statistical power of QSART as an endpoint measure.

IENFD is more sensitive in detecting early diabetic neuropathy than electrophysiologic tests and maintains excellent reproducibility when biopsies are evaluated by separate readers $(\mathrm{ICC}=0.98)$ and separate punch specimens are evaluated (at the same site; $r=0.87) . .^{20,24,25,30,34,43,48}$ The advantage of continuing to use electrophysiologic and psychophysical tests is that they are non-invasive, commonly available in many clinical trial centers, and provide rapid assessment of neuropathy based on standardized approaches to diagnosis. QST is a highly reproducible test when it is used in a multicenter trial, and reproducibility was similar in both participating sites in this study, whereas this was not true for QSART.

The main limitation of this study was the small sample size. As mentioned earlier, the similar results obtained for QST testing to other reported studies for intratrial reproducibility suggest that this was not a significant factor. The significantly low intrasession reproducibility and lower reproducibility of the mean of session 1 vs. session 2 may have been due to the small sample size. A benefit of this study is that the studies were performed by one technician at each site, and that the results were blinded to the investigators, minimizing bias.

In conclusion, QST using the CASE IV device would be an appropriate screening tool and endpoint measure for multicenter trials of neuropathy in IGR. QSART, using the QSWEAT device, has only moderate reproducibility, which reduces its value as an endpoint measure.

This study was supported in part by NIH T32 NS07222 (to A.C.P.); NIH NS40458 and DK064814 (to J.R.S., J.H., G.S.); NIH NS40458 (to J.G.); NIH NS40458, NS36778, NIH NS38849, PFUND, and JDRF (to E.L.F); NIH NS40458, NS42056, JDRF, Office of Re- search Development (Rehabilitation and Biomedical Research Service), Department of Veterans Affairs, and ADA (to J.W.R.); MO1-RR00042 (Michigan GCRC); DK02-016 (Utah GCRC); NIDDK 5P60DK-20572 (MDRTC). The authors thank Dr. Phillip Low, Mayo Clinic, Rochester, Minnesota, for assistance with analysis of the QSART data, and Susan Nalepa for technical assistance during the trial.

\section{REFERENCES}

1. Addeo R, Caraglia M, Costanzo R, Faiola V, Montella L, Abbruzzese A, et al. Oxaliplatin/rituximab combination in the treatment of intermediate-low grade non-Hodgkin's lymphoma of elderly patients. Oncol Rep 2004;12:135-140.

2. Adriaensen H, Gybels J, Handwerker HO, Van HJ. Response properties of thin myelinated (A-delta) fibers in human skin nerves. J Neurophysiol 1983;49:111-122.

3. Apfel SC, Schwartz S, Adornato BT, Freeman R, Biton V, Rendell M, et al. Efficacy and safety of recombinant human nerve growth factor in patients with diabetic polyneuropathy: a randomized controlled trial. JAMA 2000;284:2215-2221.

4. Bird SJ, Brown MJ, Spino C, Watling S, Foyt HL. Value of repeated measures of nerve conduction and quantitative sensory testing in a diabetic neuropathy trial. Muscle Nerve 2006; $34: 214-224$.

5. Bril V, Kojic J, Ngo M, Clark K. Comparison of a neurothesiometer and vibration in measuring vibration perception thresholds and relationship to nerve conduction studies. Diabetes Care 1997;20:1360-1362.

6. Cohen JA, Jeffers BW, Faldut D, Marcoux M, Schrier RW. Risks for sensorimotor peripheral neuropathy and autonomic neuropathy in non-insulin-dependent diabetes mellitus (NIDDM). Muscle Nerve 1998;21:72-80.

7. Dyck PJ, Davies JL, Litchy WJ, O'Brien PC. Longitudinal assessment of diabetic polyneuropathy using a composite score in the Rochester Diabetic Neuropathy Study cohort. Neurology 1997;49:229-239.

8. Low PA, Denq JC, Opfer-Gehrking TL, Dyck PJ, O'Brien PC, Slezak JM. Effect of age and gender on sudomotor and cardiovagal function and blood pressure response to tilt in normal subjects. Muscle Nerve 1997;20:1561-1568.

9. Dyck PJ, Dyck PJ, Kennedy WR, Kesserwani H, Melanson M, Ochoa J, et al. Limitations of quantitative sensory testing when patients are biased toward a bad outcome. Neurology 1998;50:1213.

10. Dyck PJ, Karnes JL, O’Brien PC, Litchy WJ, Low PA, Melton LJ III. The Rochester Diabetic Neuropathy Study: reassessment of tests and criteria for diagnosis and staged severity. Neurology 1992;42:1164-70.

11. Dyck PJ, Kratz KM, Karnes JL, Litchy WJ, Klein R, Pach JM, et al. The prevalence by staged severity of various types of diabetic neuropathy, retinopathy, and nephropathy in a population-based cohort: the Rochester Diabetic Neuropathy Study. Neurology 1993;43:817-824.

12. Dyck PJ, O'Brien PC, Kosanke JL, Gillen DA, Karnes JL. A 4, 2 , and 1 stepping algorithm for quick and accurate estimation of cutaneous sensation threshold. Neurology 1993;43:15081512.

13. Dyck PJ, Zimmerman I, Gillen DA, Johnson D, Karnes JL, O'Brien PC. Cool, warm, and heat-pain detection thresholds: testing methods and inferences about anatomic distribution of receptors. Neurology 1993;43:1500-1508.

14. England JD, Gronseth GS, Franklin G, Miller RG, Asbury AK, Carter GT, et al. Distal symmetrical polyneuropathy: definition for clinical research. Muscle Nerve 2005;31:113-123.

15. Freeman R, Chase KP, Risk MR. Quantitative sensory testing cannot differentiate simulated sensory loss from sensory neuropathy. Neurology 2003;60:465-470. 
16. Genuth S, Alberti KG, Bennett P, Buse J, Defronzo R, Kahn R, et al. Follow-up report on the diagnosis of diabetes mellitus. Diabetes Care 2003;26:3160-3167.

17. Grandinetti A, Chow DC, Sletten DM, Oyama JK, Theriault AG, Schatz IJ, et al. Impaired glucose tolerance is associated with postganglionic sudomotor impairment. Clin Auton Res 2007;17:231-233.

18. Hallin RG, Torebjork HE, Wiesenfeld Z. Nociceptors and warm receptors innervated by $\mathrm{C}$ fibres in human skin. J Neurol Neurosurg Psychiatry 1982;45:313-319.

19. Kastenbauer T, Sauseng S, Brath H, Abrahamian H, Irsigler K. The value of the Rydel-Seiffer tuning fork as a predictor of diabetic polyneuropathy compared with a neurothesiometer. Diabet Med 2004;21:563-567.

20. Kennedy WR, Wendelschafer-Crabb G. Utility of the skin biopsy method in studies of diabetic neuropathy. Electroencephalogr Clin Neurophysiol 1999;50(suppl):553-559.

21. Kihara M, Mitsui M, Nishikawa S, Nishimoto K, Takahashi M. Comparison of electrophysiologic and autonomic tests in sensory diabetic neuropathy. Clin Auton Res 1998;8:213-220.

22. Kincaid JC, Price KL, Jimenez MC, Skljarevski V. Correlation of vibratory quantitative sensory testing and nerve conduction studies in patients with diabetes. Muscle Nerve 2007;36:821827.

23. Lehtinen JM, Niskanen L, Hyvonen K, Siitonen O, Uusitupa M. Nerve function and its determinants in patients with newlydiagnosed type 2 (non-insulin-dependent) diabetes mellitus and in control subjects-a 5-year follow-up. Diabetologia 1993;36:68-72.

24. Levy DM, Karanth SS, Springall DR, Polak JM. Depletion of cutaneous nerves and neuropeptides in diabetes mellitus: an immunocytochemical study. Diabetologia 1989;32:427-433.

25. Lindberger M, Schroder HD, Schultzberg M, Kristensson K, Persson A, Ostman J, et al. Nerve fibre studies in skin biopsies in peripheral neuropathies. I. Immunohistochemical analysis of neuropeptides in diabetes mellitus. J Neurol Sci 1989;93: 289-296.

26. Low PA, Caskey PE, Tuck RR, Fealey RD, Dyck PJ. Quantitative sudomotor axon reflex test in normal and neuropathic subjects. Ann Neurol 1983;14:573-580.

27. Low PA, Opfer-Gehrking TL, Dyck PJ, Litchy WJ, O'Brien PC. Double-blind, placebo-controlled study of the application of capsaicin cream in chronic distal painful polyneuropathy. Pain 1995;62:163-168.

28. Lowenstein L, Jesse K, Kenton K. Comparison of perception threshold testing and thermal-vibratory testing. Muscle Nerve 2008;37:514-517.

29. Luciano CA, Russell JW, Banerjee TK, Quirk JM, Scott LJ, Dambrosia JM, et al. Physiological characterization of neuropathy in Fabry's disease. Muscle Nerve 2002;26:622-629.

30. McArthur JC, Stocks EA, Hauer P, Cornblath DR, Griffin JW. Epidermal nerve fiber density: normative reference range and diagnostic efficiency. Arch Neurol 1998;55:1513-1520.

31. Novak V, Kanard R, Kissel JT, Mendell JR. Treatment of painful sensory neuropathy with tiagabine: a pilot study. Clin Auton Res 2001;11:357-361.

32. Novella SP, Inzucchi SE, Goldstein JM. The frequency of undiagnosed diabetes and impaired glucose tolerance in pa- tients with idiopathic sensory neuropathy. Muscle Nerve 2001; 24:1229-1231.

33. Periquet MI, Novak V, Collins MP, Nagaraja HN, Erdem S, Nash SM, et al. Painful sensory neuropathy: prospective evaluation using skin biopsy. Neurology 1999;53:1641-1647.

34. Polydefkis M, Hauer P, Griffin JW, McArthur JC. Skin biopsy as a tool to assess distal small fiber innervation in diabetic neuropathy. Diabetes Technol Ther 2001;3:23-28.

35. Reading P, Russell JW. Chronic autonomic neuropathies. In: Gilman S, editor. Medlink, the information resource for clinical neurology. La Jolla, CA: Arbor; 2005.

36. Report of the Expert Committee on the Diagnosis and Classification of Diabetes Mellitus. Diabetes Care 1997;20:11831197.

37. Russell JW. Quantitative sensory testing. In: Bromberg M, Smith AG, editors. Handbook of peripheral neuropathy. London: Taylor and Francis; 2005. p. 45-52.

38. Russell JW, Feldman EL. Impaired glucose tolerance-does it cause neuropathy? Muscle Nerve 2001;24:1109-1112.

39. Schifitto G, Yiannoutsos C, Simpson DM, Adornato BT, Singer EJ, Hollander $\mathrm{H}$, et al. Long-term treatment with recombinant nerve growth factor for HIV-associated sensory neuropathy. Neurology 2001;57:1313-1316.

40. Shrout PE, Fleiss JL. Intraclass correlations: uses in assessing rater reliability. Psychol Bull 2008;86:420-427.

41. Singleton JR, Smith AG, Bromberg MB. Painful sensory polyneuropathy associated with impaired glucose tolerance. Muscle Nerve 2001;24:1225-1228.

42. Singer W, Spies JM, McArthur J, Low J, Griffin JW, Nickander $\mathrm{KK}$, et al. Prospective evaluation of somatic and autonomic small fibers in selected autonomic neuropathies. Neurology 2004;62:612-618

43. Smith AG, Howard JR, Kroll R, Ramachandran P, Hauer P, Singleton JR, et al. The reliability of skin biopsy with measurement of intraepidermal nerve fiber density. J Neurol Sci 2005; 228:65-69.

44. Singleton JR, Smith AG, Russell JW, Feldman EL. Microvascular complications of impaired glucose tolerance. Diabetes 2003;52:2867-2873.

45. Smith AG, Ramachandran P, Tripp S, Singleton JR. Epidermal nerve innervation in impaired glucose tolerance and diabetesassociated neuropathy. Neurology 2001;57:1701-1704.

46. Smith AG, Russell J, Feldman EL, Goldstein J, Peltier A, Smith $\mathrm{S}$, et al. Lifestyle intervention for pre-diabetic neuropathy. Diabetes Care 2006;29:1294-1299.

47. Sumner CJ, Sheth S, Griffin JW, Cornblath DR, Polydefkis M. The spectrum of neuropathy in diabetes and impaired glucose tolerance. Neurology 2003;60:108-111.

48. Vlckova-Moravcova E, Bednarik J, Dusek L, Toyka KV, Sommer C. Diagnostic validity of epidermal nerve fiber densities in painful sensory neuropathies. Muscle Nerve 2008;37:5060.

49. Ziegler D, Gries FA, Spuler M, Lessmann F. The epidemiology of diabetic neuropathy. Diabetic Cardiovascular Autonomic Neuropathy Multicenter Study Group. J Diabet Complic 1992; $6: 49-57$. 